\title{
Impact of Cosmic Rays on Stratospheric Chlorine Chemistry and Ozone Depletion
}

\author{
Rolf Müller* \\ ICG-I, Research Centre Jülich, 52425 Jülich, Germany
}

(Received 13 March 2003; published 31 July 2003)

\begin{abstract}
Dissociation induced by cosmic rays of chlorofluorocarbons (CFC) and $\mathrm{HCl}$ on the surfaces of polar stratospheric clouds (PSC) has been suggested as playing a significant role in causing the ozone hole. However, observed stratospheric CFC distributions are inconsistent with a destruction of CFC on PSC surfaces and no significant correlation exists between ozone levels and cosmic-ray activity inside the polar regions. Model simulations indicate that this mechanism can have only a limited impact on chemical ozone loss and thus on the recovery of stratospheric ozone.
\end{abstract}

DOI: 10.1103/PhysRevLett.91.058502

PACS numbers: 92.70.Cp, 82.33.Tb, 94.10.Fa

In the Earth's atmosphere, below $\approx 70 \mathrm{~km}$, cosmic rays are the primary source of ionization. The cosmic-rayinduced ionization causes the production of radicals $(\mathrm{NO}$ and $\mathrm{OH})$ that play a role in the stratospheric polar ozone chemistry [1,2]. Recently, Lu and Sanche [3-6] suggested that the action of cosmic rays on polar stratospheric clouds (PSC) causes rapid destruction of chlorofluorocarbons (CFC) and $\mathrm{HCl}$, and that this process may constitute a new pathway for the formation of the "ozone hole." Ozone hole is the term commonly used to refer to the very strong depletion of the total ozone column that has been observed since the early eighties in the Antarctic spring [7-9]. Similarly, since the early 1990s, substantial losses of total column ozone have been observed in cold and protracted Arctic winters [8-10].

$\mathrm{Lu}$ and Sanche [3] found that ozone loss is strongly correlated with cosmic-ray ionization-rate variations with altitude, latitude, and solar cycle. They suggest that the physical basis of these observations is dissociative electron attachment (DEA) to CFC on the surface of PSC particles, due to cosmic-ray radiation that causes CFC-11 and $\mathrm{CFC}-12$ to be rapidly destroyed in the winter polar stratosphere at altitudes below $20 \mathrm{~km}$.

This view has been criticized by the argument that the observed CFC distribution in the stratosphere can be explained by known transport and chemical processes $[11,12]$. Furthermore, it was questioned whether a correlation actually exists between the observed ozone loss and cosmic-ray activity [11] and what the impact of DEAinduced degradation of CFC would be on estimates of the recovery of the ozone hole [12]. Here, the question of a relation between PSC occurrence, cosmic-ray flux, CFC degradation, and stratospheric ozone depletion is revisited. For this purpose, the DEA-induced heterogeneous chemistry of both $\mathrm{CFC}$ [3] and $\mathrm{HCl}$ [4] was implemented in a numerical photochemical model of the stratosphere [13].

$\mathrm{Lu}$ and Sanche [3] note that the altitudinal and latitudinal dependence of the observed polar ozone losses is in accordance with the established patterns of the intensity of cosmic-ray ionization rates. However, the same is true, and much more strictly, for the patterns of occurrence of PSC that are observed only in the winter polar stratosphere $[8,9,14]$. That is, both the altitudinal and the latitudinal patterns of strong ozone losses in the polar regions are controlled by the occurrence of PSC $[8,9,14]$. Since both the conventional and the $\mathrm{Lu}$ and Sanche [3] pathway for the formation of the ozone hole require the presence of PSC, no distinction between the two pathways is possible through this observation.

However, the observed rate of ozone loss from 1979 to 1998 over northern midlatitudes $\left(40^{\circ}-53^{\circ} \mathrm{N}\right)$ clearly cannot be due to the occurrence of PSC [Fig. 1(b) of Ref. [3] ]. Further, the latitudinal variation of cosmic-ray intensity between the pole and the equator is only about a factor of $2[3,15]$ compared to a much stronger latitudinal gradient in both PSC occurrence and ozone depletion [8,9]. Therefore, the latitudinal variation of strong ozone depletion is most likely caused by the specific conditions inside the air mass confined in the polar vortex $[8,9]$.

$\mathrm{Lu}$ and Sanche [3] further suggest that the annual average ozone for latitudes $0^{\circ}-65^{\circ} \mathrm{S}$ over the period 1979-1992 exhibits a variation in inverse phase with cosmic-ray activity. The correlation between the cosmic-ray intensity and the ozone trend in this latitude region [from Fig. 3 of Ref. [3]] is -0.35 or, after removing a linear negative trend, -0.71 . Indeed, it is well established that long-term total ozone variations in the tropics and midlatitudes possess a component that is in phase with the 11-year solar cycle (and, thus, in inverse phase with cosmic-ray activity) [16] and that upper stratospheric ozone concentrations vary with an amplitude of a few percent over a solar cycle [16,17]. However, the Lu and Sanche [3] mechanism requires the presence of PSC that only occur in the polar regions and only during winter [8,14]. Therefore, one should compare (Fig. 1) both solar activity and cosmic-ray intensity with the average total ozone value for the northern and southern hemisphere polar regions in March and October, respectively [18]. There is no apparent correlation between cosmic-ray intensity and polar ozone in the northern or southern hemisphere; correlation coefficients are -0.32 


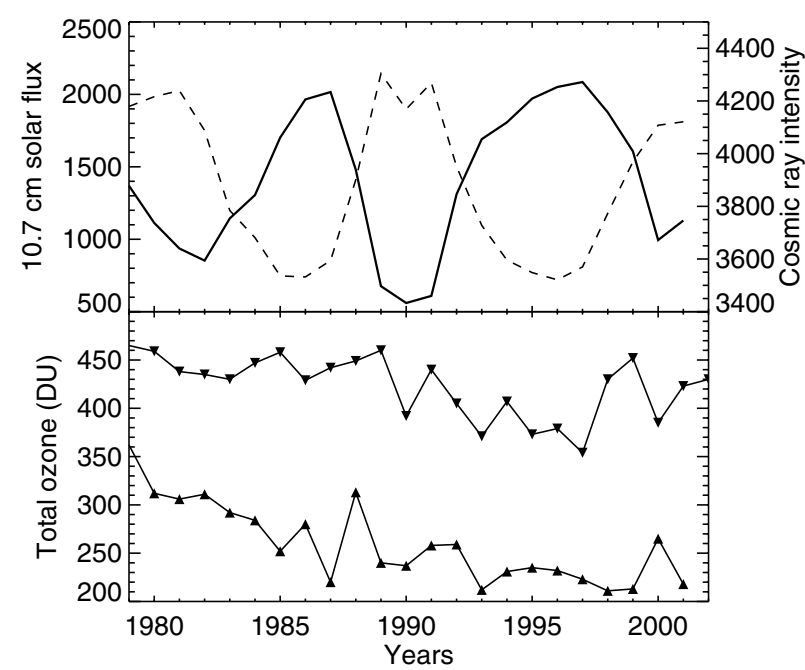

FIG. 1. The variation over the time period 1979-2001 of cosmic-ray intensity (solid line, top panel), solar intensity (dashed line, top panel), and total ozone in spring averaged over the polar region (bottom panel). The pressure-corrected neutron count at Climax, Colorado, U.S.A., is shown as a measure of cosmic-ray intensity and the $10.7 \mathrm{~cm}(2800 \mathrm{MHz})$ solar flux adjusted to 1 a.u. in units of $10^{-21} \mathrm{~W} \mathrm{~m}^{-2} \mathrm{~Hz}^{-1}$ as a measure of solar intensity. The polar total ozone values constitute averages over the latitude region $63^{\circ}-90^{\circ}$ for March (downward triangles) and October (upward triangles) in the northern and southern hemispheres, respectively. Total ozone measurements were taken from a series of TOMS satellite instruments [updated from Ref. [18]; Newman, personal communication].

and -0.34 , respectively, or, if a linear trend (of -2.4 and $-4.8 \mathrm{DU} /$ year for the northern and southern hemisphere, respectively) is removed from the time series, -0.22 and -0.20 , respectively.

Moreover, Lu and Sanche [3] conjecture that the depletion of CFC in the stratosphere is related to the combined effect of cosmic-ray radiation and the presence of PSC, but not to solar radiation. This would imply that CFC are solely depleted by the action of cosmic rays within PSC, that is, solely and extremely rapidly, with a lifetime in the order of hours, within the polar regions in winter. Such a view contrasts with observations of both substantially reduced CFC mixing ratios in the tropics in the middle stratosphere (where no PSC occur) and low, but nonzero, mixing ratios of CFC in the polar vortex in air masses that have experienced PSC processing over at least a few days [19-21].

The observed stratospheric CFC patterns $[5,21,22]$ are, however, in accordance with the conventional picture of being due to the combined effect of large-scale transport and degradation of CFC by photolysis $[11,12]$. The largescale circulation in the stratosphere is characterized by slow upward motion in the tropics, poleward and downward motion in midlatitudes, and by descent in the high latitudes [23-25]. The CFC in the stratosphere are destroyed by photolysis during upward transport in the tropics, so that mixing ratios decrease with altitude. Further, air masses at greater altitudes, characterized by low CFC mixing ratios, are transported poleward, followed by downward transport due to diabatic descent into the winter polar region. This means that by far the largest fraction of the CFC in the air within the polar winter stratosphere has already been chemically converted so that, if any additional CFC processing were to occur, it would have only a moderate impact on the lifetimes of CFC. Disagreement between observed mixing ratios of CFC-12 and simulations with two-dimensional photochemical models [5] should not be taken as an indication of missing chemical processes, but are rather caused by the limitations of the representation of transport in twodimensional models [9].

Trace gases that are destroyed by photochemical degradation, at fast rates compared to the time scales of vertical transport, show compact relations in scatter diagrams of the mixing ratios of two such trace gases against each other [26]. Deviation from such compact relations are being used for the identification of physiochemical change in the presence of trace gas variations due to transport $[10,12,19]$. Figure 2 shows an example of the compact relation of CFC-11 and CFC-12 inside the polar vortex against a chemically long-lived (and thus quasiinert over the time scales in question) tracer of stratospheric motion such as $\mathrm{N}_{2} \mathrm{O}$. Both relations are rather compact irrespective of when and where the contributing measurements were taken. Such a pattern is incompatible

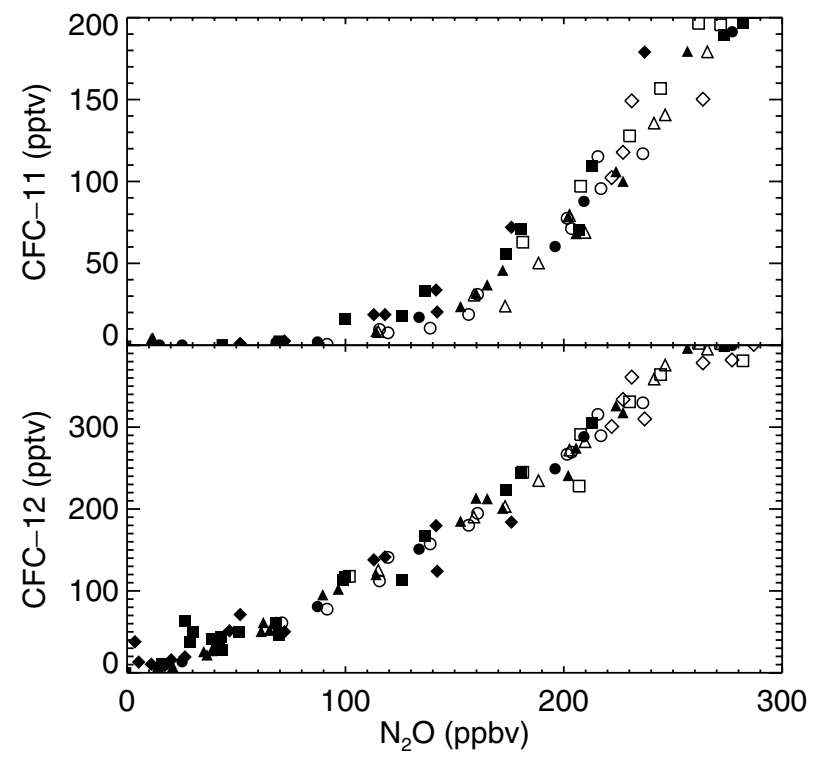

FIG. 2. Scatter diagrams of the observed mixing ratios of $\mathrm{N}_{2} \mathrm{O}$ and CFC-11 $\left(\mathrm{CFCl}_{3}\right)$ and $\mathrm{N}_{2} \mathrm{O}$ and CFC-12 $\left(\mathrm{CF}_{2} \mathrm{Cl}_{2}\right)$, top and bottom panels, respectively, in winter 1991-1992. Shown are measurements inside (solid symbols) and outside (open symbols) the polar vortex. Measurements were made in December (circles), January (triangles), February (diamonds), and March (squares). See Refs. [19,20] for detailed information on the measurements. 
with the view that the lifetime of both CFC-11 and CFC12 in PSC is in the order of hours in the presence of PSC. This should have led to extremely low concentrations of CFC-11 and CFC-12 inside the Arctic vortex during January 1992, a period that was characterized by temperatures low enough to promote strong PSC activity [27].

The DEA-induced destruction of CFC-11, CFC-12, and $\mathrm{HCl}$ on PSC was implemented in a numerical model of stratospheric chemistry [13]; it is assumed that CFC$11, \mathrm{CFC}-12$, and $\mathrm{HCl}$ are decomposed on the surfaces of PSC particles with lifetimes taken from Refs. [3,4] (Table I). These lifetimes were calculated based on the assumption that $\mathrm{CFC}-11, \mathrm{CFC}-12$, and $\mathrm{HCl}$ are adsorbed on the surface of the PSC. Without adsorption, DEAinduced destruction on PSC cannot occur [12]. On the other hand, because there are no reaction channels competing with DEA-induced loss of CFC, the results of the model calculations are not sensitive to the precise value of the assumed loss rates. The considered airmass is representative of the polar vortex in the Arctic in winter 19911992 [28]. The calculation is initialized for 15 December 1991; initial mixing ratios are as in Ref. [28], except for CFC-11 (4 pptv) and CFC-12 (80 pptv), corresponding to $\mathrm{N}_{2} \mathrm{O}=80 \mathrm{ppbv}$ (Fig. 2). The calculation is carried through until 15 March 1992; the vortex air descends from $\approx 21 \mathrm{~km}$ to $\approx 17 \mathrm{~km}$ over that time period [28]. Three cases were considered (Fig. 3): (a) a calculation including DEA-induced heterogeneous destruction of CFC-11, CFC-12, and $\mathrm{HCl}$; (b) a simulation with DEAinduced destruction only of CFC-11 and CFC-12; and (c), the standard case, a simulation without any DEA-induced heterogeneous chemistry.

Compared to the standard case (c), considering DEAinduced destruction of only CFC-11 and CFC-12 [case (b)] results in somewhat greater mixing ratios of active chlorine $\left(\mathrm{ClO}+2 \mathrm{Cl}_{2} \mathrm{O}_{2}\right)$, due to the activation of the chlorine contained in CFC-11 and CFC-12, and, consequently, a somewhat greater ozone destruction (by $\approx 8 \%$ ). However, the air in the polar vortex has been in the stratosphere long enough (typically several years) for the major fraction of CFC-11 and CFC-12 to be chemically converted to other compounds (mainly $\mathrm{HCl}$ and $\mathrm{ClONO}_{2}$ ) at the time when PSC form. Therefore, at that time the chlorine contained in CFC- 11 and CFC- 12 constitutes only a minor fraction of the atmospheric chlorine so that any additional processing can have only a minor

TABLE I. Lifetimes of CFC-11, CFC-12, and $\mathrm{HCl}$ due to DEA on PSC.

\begin{tabular}{ccccc}
\hline \hline Species & $\sigma_{\text {DEA }}\left(\mathrm{cm}^{-2}\right)$ & $R\left(\mathrm{~s}^{-1}\right)$ & $\tau(\mathrm{s})$ & $\tau(\mathrm{h})$ \\
\hline $\mathrm{CFC}-11$ & $1.1 \times 10^{-13 \mathrm{a}}$ & $3.6 \times 10^{-4} \mathrm{a}$ & $2.8 \times 10^{3}$ & 0.8 \\
$\mathrm{CFC}-12$ & $1.3 \times 10^{-14 \mathrm{a}}$ & $4.3 \times 10^{-5} \mathrm{a}$ & $2.3 \times 10^{4}$ & 6.5 \\
$\mathrm{HCl}$ & $4.0 \times 10^{-15 \mathrm{~b}}$ & $1.3 \times 10^{-5}$ & $7.7 \times 10^{4}$ & 21 \\
\hline \hline
\end{tabular}

aTaken from Ref. [3].

baken from Ref. [4]. effect on the levels of total inorganic chlorine $\left(\mathrm{Cl}_{y}\right)$ and thus on the levels of active chlorine $\left(\mathrm{ClO}_{x}\right)$.

A somewhat greater impact on the model results is given by the assumption of DEA-induced $\mathrm{HCl}$ destruction that leads to a practically complete activation of the $\mathrm{HCl}$ reservoir in case (a) compared to (b) (Fig. 3). While this does not impact $\mathrm{Cl}_{y}$, it results in initially greater active chlorine mixing ratios. However, the extremely low $\mathrm{HCl}$ mixing ratios allow a more rapid increase of $\mathrm{ClONO}_{2}$, i.e., a more rapid deactivation of chlorine starting after the last simulated PSC activity around mid-January that eventually leads to slightly lower ozone loss in case (a) compared to (b).

The major result of the model simulations is that the temporal development of the key chemical species, and, in particular, the ozone loss, does not drastically alter depending on whether loss of CFC-11, CFC-12, and $\mathrm{HCl}$

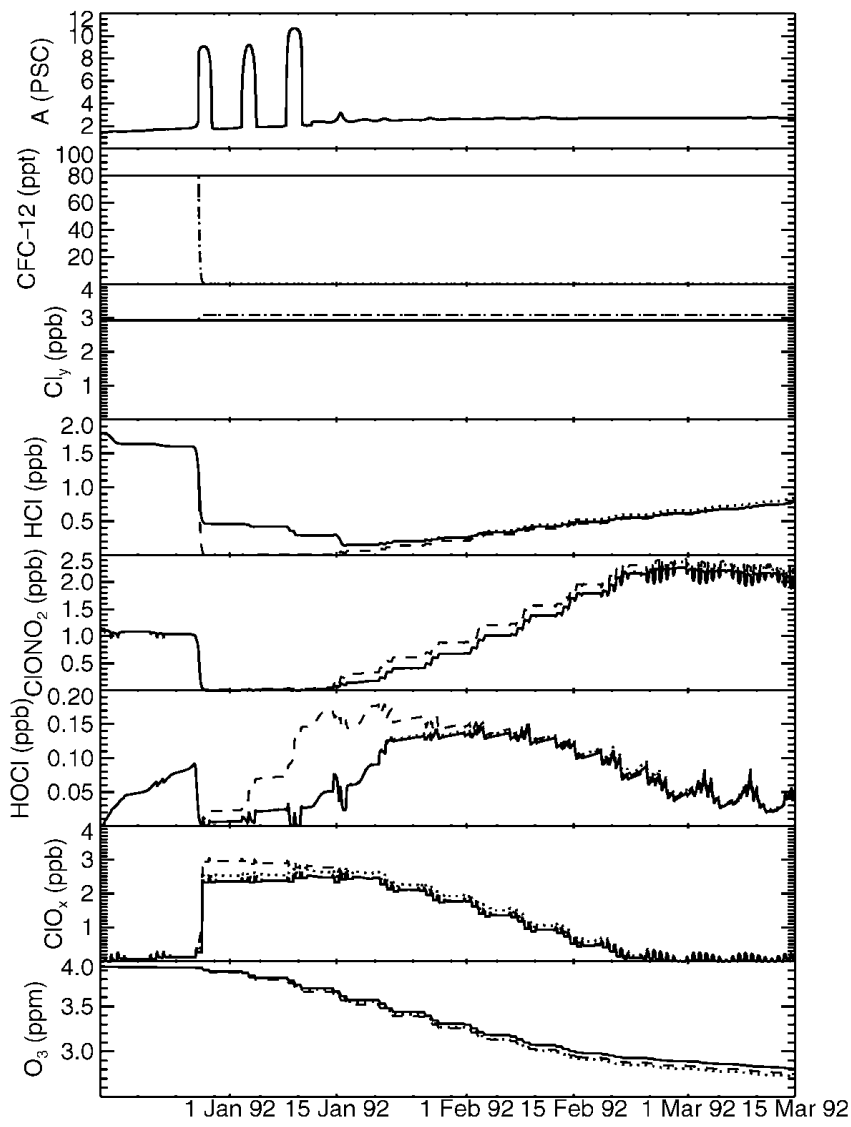

FIG. 3. Results of model simulations of an airmass representative of the Arctic polar vortex in 1991-1992. Three cases are shown: dashed line, a simulation including DEA-induced heterogeneous destruction of CFC-11, CFC-12, and $\mathrm{HCl}$ (case a); dotted line, a simulation with DEA-induced destruction only of CFC-11 and CFC-12 (case b); and, solid line, a simulation without any DEA-induced heterogeneous chemistry (case c). The top panel shows the total simulated PSC surface area density (in $\mu \mathrm{m}^{2} / \mathrm{cm}^{3}$ ), and the panels below the volume mixing ratios of key chemical species (where the active chlorine $\mathrm{ClO}_{x}$ is defined as $\mathrm{ClO}+2 \mathrm{Cl}_{2} \mathrm{O}_{2}$ and total inorganic chlorine $\mathrm{Cl}_{y}$ as $\mathrm{HCl}+\mathrm{ClONO}_{2}+\mathrm{HOCl}+\mathrm{ClO}_{x}$. 
on PSC due to DEA is included in the model or not. The only exceptions are the concentrations of CFC-11 (not shown) and CFC-12 (Fig. 3) that take on mixing ratios of practically zero within hours after the first PSC event if destruction due to DEA is included in the model. Otherwise, CFC-11 and CFC-12 are practically chemically inert in the polar winter stratosphere (Fig. 3). In the case of $\mathrm{HCl}$, DEA-induced destruction on PSC competes with the likewise very rapid heterogeneous reaction of $\mathrm{HCl}$ with $\mathrm{ClONO}_{2}$ and $\mathrm{HOCl}$ on PSC surfaces [14]. Consequently, the simulated $\mathrm{HCl}$ concentrations with and without DEA-induced $\mathrm{HCl}$ degradation do not differ substantially; only in the former case do $\mathrm{HCl}$ concentrations reach extremely low levels within hours after the first occurrence of PSC, a process that is otherwise halted by the titration of the heterogeneous reaction partners $\left(\mathrm{ClONO}_{2}\right.$ and $\left.\mathrm{HOCl}\right)$ of $\mathrm{HCl}$ (Fig. 3). The loss rate of ozone is little affected by assumptions about the occurrence of DEA-induced reactions on $\mathrm{PSC} ; \approx 5 \%$ more ozone is chemically destroyed in the model if DEAinduced loss of CFC-11, CFC-12, and $\mathrm{HCl}$ is taken into account. The rather small changes predicted for the mixing ratios of key species due to DEA-induced reactions are unlikely to be detectable by satellite measurements. However, high precision in situ measurements of CFC-11, $\mathrm{CFC}-12$, and $\mathrm{HCl}$ in an airmass shortly after the first occurrence of PSC might allow identifying the effect of DEA-induced reactions in the atmosphere.

In summary, the ozone loss mechanism proposed by $\mathrm{Lu}$ and Sanche [3] cannot be of relevance outside the polar regions in winter, where PSC do not occur $[8,9,14]$. Within the polar regions, there is no significant correlation between ozone levels and cosmic-ray activity (Fig. 1). Further, the observed spatial distributions of CFC-11 and CFC-12 in the stratosphere are inconsistent with the notion that these compounds are exclusively, and very rapidly, destroyed by DEA on PSC particles. Moreover, model simulations indicate that stratospheric polar ozone chemistry - most notably chemical ozone destructionis not substantially altered if DEA-induced processes on PSC are taken into account (Fig. 3). However, DEAinduced destruction of $\mathrm{HCl}$ on PSC could have some influence on stratospheric chlorine chemistry. At this point, it seems an open question whether or not the latter process occurs in the atmosphere. In any case, the impact of DEA processes on ozone recovery is expected to be small because of their limited influence on both CFC lifetimes and on chemical ozone loss.

Thanks are due to P. Newman for providing the TOMS total ozone data. The cosmic-ray intensities were obtained from the WDC-C2 Data Center for Cosmic Rays at Ibaraki University (http://www.env.sci.ibaraki.ac.jp/ database/html/WDCCR/wdccr_e.html). The $10.7 \mathrm{~cm}$ solar flux was obtained via the Coupling, Energetics and Dynamics of Atmospheric Regions (CEDAR) program (http://cedarweb.hao.ucar.edu/instr/gpi.html). I would also like to thank U. Kellner and A. Krimpmann for their help with the data analysis and B. Vogel for pointing out to me Ref. [3].

*Electronic address: ro.mueller@fz-juelich.de URL: http://www.fz-juelich.de/icg/icg-i/www_export/ user/ro.mueller/index.html

[1] R. Müller and P. J. Crutzen, J. Geophys. Res. 98, 20483 (1993).

[2] P. J. Crutzen and F. Arnold, Nature (London) 342, 651 (1986).

[3] Q.-B. Lu and L. Sanche, Phys. Rev. Lett. 87, 078501 (2001).

[4] Q.-B. Lu and L. Sanche, J. Chem. Phys. 115, 5711 (2001).

[5] Q.-B. Lu and L. Sanche, Phys. Rev. Lett. 89, 219802 (2002).

[6] Q.-B. Lu and L. Sanche, Phys. Rev. Lett. 89, 219804 (2002).

[7] A. E. Jones and J. D. Shanklin, Nature (London) 376, 409 (1995).

[8] S. Solomon, Rev. Geophys. 37, 275 (1999).

[9] WMO, Report No. 44, Geneva, 1998.

[10] R. Müller, P. J. Crutzen, J.-U. Grooß, C. Brühl, J. M. Russell III, H. Gernandt, D. S. McKenna, and A. F. Tuck, Nature (London) 389, 709 (1997).

[11] P. K. Patra and M. Santhanam, Phys. Rev. Lett. 89, 219803 (2002).

[12] N. R. P. Harris, J. Farman, and D. Fahey, Phys. Rev. Lett. 89, 219801 (2002).

[13] D. S. McKenna, J.-U. Grooß, G. Günther, P. Konopka, R. Müller, G. Carver, and Y. Sasano, J. Geophys. Res. 107, 10.1029/2000JD000113 (2002).

[14] T. Peter, Annu. Rev. Phys. Chem. 48, 785 (1997).

[15] P. Grieder, Cosmic Rays at Earth (Elsevier, Amsterdam, 2001).

[16] S. Chandra and R. D. McPeters, J. Geophys. Res. 99, 20665 (1994).

[17] A. Miller et al., J. Geophys. Res. 102, 19257 (1997).

[18] P. Newman, F. Gleason, R. McPeters, and R. Stolarski, Geophys. Res. Lett. 24, 2689 (1997).

[19] R. Müller, U. Schmidt, A. Engel, D. McKenna, and M. Proffitt, Q. J. R. Meteorol. Soc. 127, 1389 (2001).

[20] U. Schmidt, R. Bauer, A. Engel, R. Borchers, and J. Lee, Geophys. Res. Lett. 21, 1215 (1994).

[21] M. Riese, X. Tie, G. Brasseur, and D. Offermann, J. Geophys. Res. 104, 16419 (1999).

[22] A. E. Roche, R. W. Nightingale, J. B. Kumer, J. L. Mergenthaler, C. H. Jackman, and E. L. Fleming, Adv. Space Res. 21, 1383 (1998).

[23] G. M. B. Dobson, D. Harrison, and J. Lawrence, Proc. R. Soc. London, Ser. A 122, 456 (1929).

[24] A.W. Brewer, Q. J. R. Meteorol. Soc. 75, 351 (1949).

[25] J. R. Holton, P. Haynes, M. E. McIntyre, A. R. Douglass, R. B. Rood, and L. Pfister, Rev. Geophys. 33, 403 (1995).

[26] R. A. Plumb and M. K. W. Ko, J. Geophys. Res. 97, 10145 (1992).

[27] J. C. Farman, A. O'Neill, and R. Swinbank, Geophys. Res. Lett. 21, 1195 (1994).

[28] G. Becker, R. Müller, D. S. McKenna, M. Rex, and K. S. Carslaw, Geophys. Res. Lett. 25, 4325 (1998). 